\title{
Synthesis and Star/Linear Topology Transformation of Mechanically Linked ABC Terpolymer
}

Hiroki Sato, ${ }^{\dagger}$ Daisuke Aoki, ${ }^{\dagger}$ and Toshikazu Takata*, ${ }^{\dagger, \dagger}$

${ }^{\dagger}$ Department of Chemical Science and Engineering and ${ }^{\ddagger}$ ST-CREST, Tokyo Institute of

Technology, O-okayama 2-12-1(H-126), Meguro-ku, Tokyo 152-8552, Japan.

ttakata@polymer.titech.ac.jp

Supporting Information

\section{Table of Contents}

$\begin{array}{lr}\text { Materials and Instruments } & \text { S2 }\end{array}$

$\begin{array}{lr}\text { Syntheses of axle (1) and wheel (2) components } & \text { S3 }\end{array}$

$\begin{array}{lr}\text { Syntheses of polymers } & \text { S6 }\end{array}$

$\begin{array}{lr}\text { Characterization of polymers } & \text { S10 }\end{array}$

$\begin{array}{lr}\text { References } & \text { S10 }\end{array}$ 


\section{Materials}

Dichloromethane was purchased from ASAHI GLASS CO., LTD., and distilled over $\mathrm{CaH}_{2}$ after being washed with water. $\delta$-Valerolactone $(\delta$-VL $)(>98 \%$, Tokyo Kasei Kogyo Co., Ltd. (TCI)) was distilled over $\mathrm{CaH}_{2}$ under reduced pressure. Styrene was used after passing through alumina column. Copper(I) bromide ( $>99 \%$, Wako Pure Chemical Industries, Ltd. (Wako)) was used after washing with conc. hydrobromic acid. 4-hydroxy-3,5-dimethylbenzaldehyde (S1) ( $>98 \%$, TCI), propargyl bromide ( $>97 \%$, TCI), lithium aluminium hydride ( $>80 \%$, Wako), 1-ethyl-3-(3-dimethylaminopropyl)carbodiimide hydrochloride (EDCI·HCl) $(>98 \%$, TCI), 4-(dimethylamino)pyridine (DMAP) (>99\%, TCI), diphenyl phosphate (DPP) (>99\%, TCI), 3,5-bis(trifluoromethyl)phenyl isocyanate ( $>98 \%$, TCI), toluene $(>99 \%$, Wako), 4-cyano-4-[(dodecylsulfanylthiocarbonyl)sulfanyl]pentanoic acid (TTC-CO $\left.\mathbf{O}_{2} \mathbf{H}\right) \quad(>97 \%$, Sigma-Aldrich $\quad$ Co.), 2,2'-azodiisobutyronitrile $\quad(A I B N) \quad(>98 \%, \quad$ TCI), $N, N, N^{\prime}, N^{\prime \prime}, N^{\prime \prime}$-pentamethyldiethylenetriamine (PMDETA) $(99 \%, \mathrm{TCI})$, acetic anhydride (>93\%, Wako), triethylamine ( $>99 \%$, Wako) and tetrahydrofuran (THF) ( $>99 \%$, anhydrous, Wako) were used as received. 12-aminododecanoate hydrochloride ${ }^{1}$, wheel-OH ${ }^{2}$ and azide-terminated PEO $^{3}$ were prepared according to literatures. Other commercially available regents and solvents were used as received.

\section{Instruments}

${ }^{1} \mathrm{H}(300 \mathrm{MHz})$ and ${ }^{13} \mathrm{C}(75 \mathrm{MHz}) \mathrm{NMR}$ spectra were recorded on a Bruker Biospin AVANCE DPX-300 spectrometer and ${ }^{1} \mathrm{H}(400 \mathrm{MHz})$ NMR spectrum was recorded on a JEOL AL-400 spectrometer, using $\mathrm{CDCl}_{3}$ as the solvent, calibrated using tetramethylsilane $\left(\delta_{\mathrm{H}}=0.00 \mathrm{ppm}\right), \mathrm{CDCl}_{3}\left(\delta_{\mathrm{C}}=77.0 \mathrm{ppm}\right)$ and DMSO- $d_{6}\left(\delta_{\mathrm{C}}=39.5 \mathrm{ppm}\right)$ as internal standards. Gel permeation chromatography (GPC) was performed at $30{ }^{\circ} \mathrm{C}$ in $\mathrm{CHCl}_{3}(0.85 \mathrm{~mL} / \mathrm{min})$ using a JASCO PU-2080 system equipped with a set of Shodex K-804 and Shodex K-805 columns. The number average molecular weight $\left(M_{\mathrm{n}}\right)$, weight average molecular weight $\left(M_{\mathrm{w}}\right)$, and polydispersity index $\left(M_{\mathrm{w}} / M_{\mathrm{n}}\right)$ of the polymers were calculated on the basis of a polystyrene calibration. Preparative GPC was carried out using a HPLC LC-918 instrument by Japan Analytical Industry with a Megapak-Gel 201C. MALDI-TOF mass spectra were taken on a Shimadzu AXIMA-CFR mass spectrometer. The spectrometer was equipped with a nitrogen laser $(1=337 \mathrm{~nm})$ and with pulsed ion extraction. The operation was performed at an accelerating potential of $20 \mathrm{kV}$ by a linear-positive ion mode. The sample polymer solution $(1 \mathrm{mg} / \mathrm{mL})$ was prepared in $\mathrm{CHCl}_{3}$, and the matrix, dithranol, and cationizing agent, sodium trifluoroacetate, were dissolved in $\mathrm{CHCl}_{3}$ or $\mathrm{CH}_{3} \mathrm{CN}$ (20 and 1 $\mathrm{mg} / \mathrm{mL}$, respectively), and $50 \mu \mathrm{L}$ of each solution were mixed prior to MALDI analysis. Thermogravimetric analyses (TGA) were performed with a Shimadzu DTG-60 instruments under nitrogen (heating rate of $10{ }^{\circ} \mathrm{C} \mathrm{min}^{-1}$ ). 
Synthesis of axle component 1
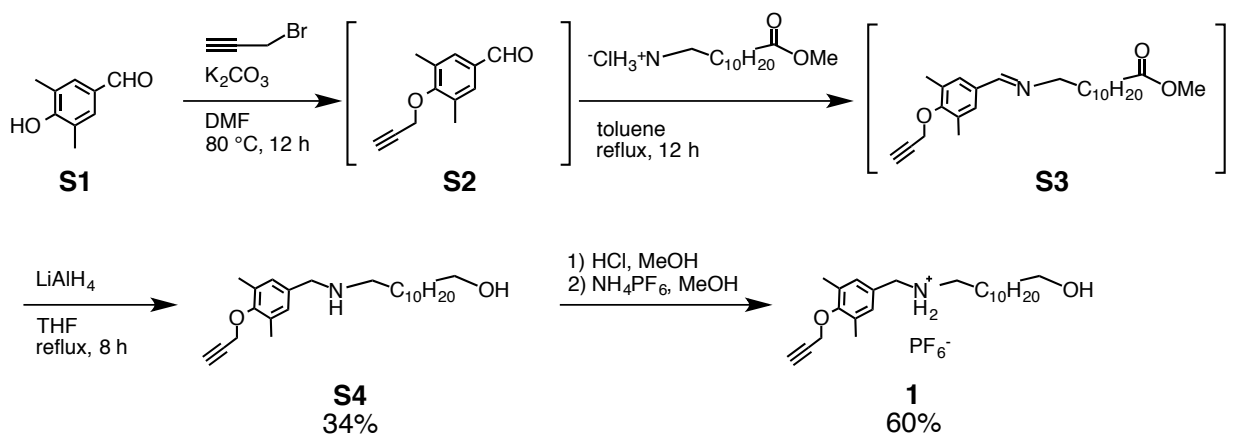

To a solution of $\mathbf{S 1}(10.0 \mathrm{~g}, 67 \mathrm{mmol})$ and propargyl bromide $(7.0 \mathrm{~mL}, 81 \mathrm{mmol})$ in DMF $(100 \mathrm{~mL})$ was added potassium carbonate $(27.6 \mathrm{~g}, 0.20 \mathrm{~mol})$ and heated at $80^{\circ} \mathrm{C}$. After $12 \mathrm{~h}$, solvent was removed under reduced pressure and the residue was diluted with chloroform followed by washing with sat. $\mathrm{NaHCO}_{3}$ and brine. The organic layer was dried over $\mathrm{MgSO}_{4}$ followed by evaporation to afford $\mathbf{S 2}$ as a brown solid, which was used without further purification.

A mixture of $\mathbf{S 2}(8.00 \mathrm{~g}, 43 \mathrm{mmol})$, methyl 12-aminododecanoate hydrochloride (12.0 g, $45 \mathrm{mmol})$ and toluene $(300 \mathrm{~mL})$ was refluxed with a Dean-Stark trap. After $12 \mathrm{~h}$, solvent was removed under reduced pressure to afford $\mathbf{S 3}$ as a brown solid, which was used without further purification.

To a solution of S3 (26.8 g, $67 \mathrm{mmol})$ in anhydrous THF (300 mL) was added dropwise a suspension of lithium aluminum hydride $(10.0 \mathrm{~g}, 0.26 \mathrm{~mol})$ in anhydrous $\mathrm{THF}(1.5 \mathrm{~L})$ at $0{ }^{\circ} \mathrm{C}$, followed by heating temperature to reflux. After $8 \mathrm{~h}$, the reaction was quenched by sat. $\mathrm{Na}_{2} \mathrm{SO}_{4}$ at $0{ }^{\circ} \mathrm{C}$. The precipitates were filtered off and the filtrate was evaporated to afford the crude product. Silica column chromatography (eluent: ethyl acetate) yielded S4 (8.40 g, 34\%) as a brown solid.

${ }^{1} \mathrm{H}-\mathrm{NMR}\left(400 \mathrm{MHz}, 298 \mathrm{~K}, \mathrm{CDCl}_{3}\right): 6.98$ (2H, s), 4.50 (2H, d, $\left.2.4 \mathrm{~Hz}\right), 3.68$ (2H, s), 3.65 (2H, t, $\left.6.8 \mathrm{~Hz}\right)$, $2.63(2 \mathrm{H}, \mathrm{t}, 6.8 \mathrm{~Hz}), 2.52(1 \mathrm{H}, \mathrm{t}, 2.4 \mathrm{~Hz}), 2.32(6 \mathrm{H}, \mathrm{s}), 1.85-1.20(20 \mathrm{H}, \mathrm{m})$.

To a solution of $\mathbf{S 4}(8.40 \mathrm{~g}, 24 \mathrm{mmol})$ in the least amount of methanol for solvation was added conc. $\mathrm{HCl}$ aq. (10.0 mL, $0.12 \mathrm{~mol})$ at ambient temperature. The mixture was stirred for a few minutes and was poured into a large amount of diethyl ether. The precipitates were collected by filtration and dried in vacuo. To a solution of the obtained precipitates in the least amount of methanol for solvation was added sat. $\mathrm{PF}_{6} \mathrm{NH}_{4}$ aq. until the precipitates were formed. The precipitates were collected by filtration, followed by washing with water and drying in vacuo to afford $1(7.59 \mathrm{~g}, 60 \%)$ as a brown solid.

${ }^{1} \mathrm{H}-\mathrm{NMR}\left(400 \mathrm{MHz}, 298 \mathrm{~K}, \mathrm{CDCl}_{3}\right): 7.07$ (2H, s, e), 6.57 (brs, - $\mathrm{NH}_{2}^{+}-$), $4.52(2 \mathrm{H}, \mathrm{d}, 2.4 \mathrm{~Hz}, \mathrm{~g}), 4.14$ $(2 \mathrm{H}, \mathrm{s}, \mathrm{d}), 3.62(2 \mathrm{H}, \mathrm{t}, 6.6 \mathrm{~Hz}, \mathrm{a}), 2.95(2 \mathrm{H}, \mathrm{brt}, 7.7 \mathrm{~Hz}, \mathrm{c}), 2.51(1 \mathrm{H}, \mathrm{t}, 2.4 \mathrm{~Hz}, \mathrm{~h}), 2.33$ (6H, s, f), 1.751.20 (20H, m, b). 
${ }^{13} \mathrm{C}-\mathrm{NMR}$ (75 MHz, $298 \mathrm{~K}$, DMSO-d $\left.{ }_{6}\right): 155.27,131.18,130.33,127.68,79.70,78.02,60.72,59.56,49.68$, $46.70,29.10,29.00,28.97,28.93,28.80,28.48,25.89,25.52,25.35,16.32$.

HR-MS (FAB ${ }^{+}$): Calcd for $\mathrm{C}_{23} \mathrm{H}_{42} \mathrm{NO}_{3}[\mathrm{M}]^{+}: 374.3059$. Found: 374.3062 .

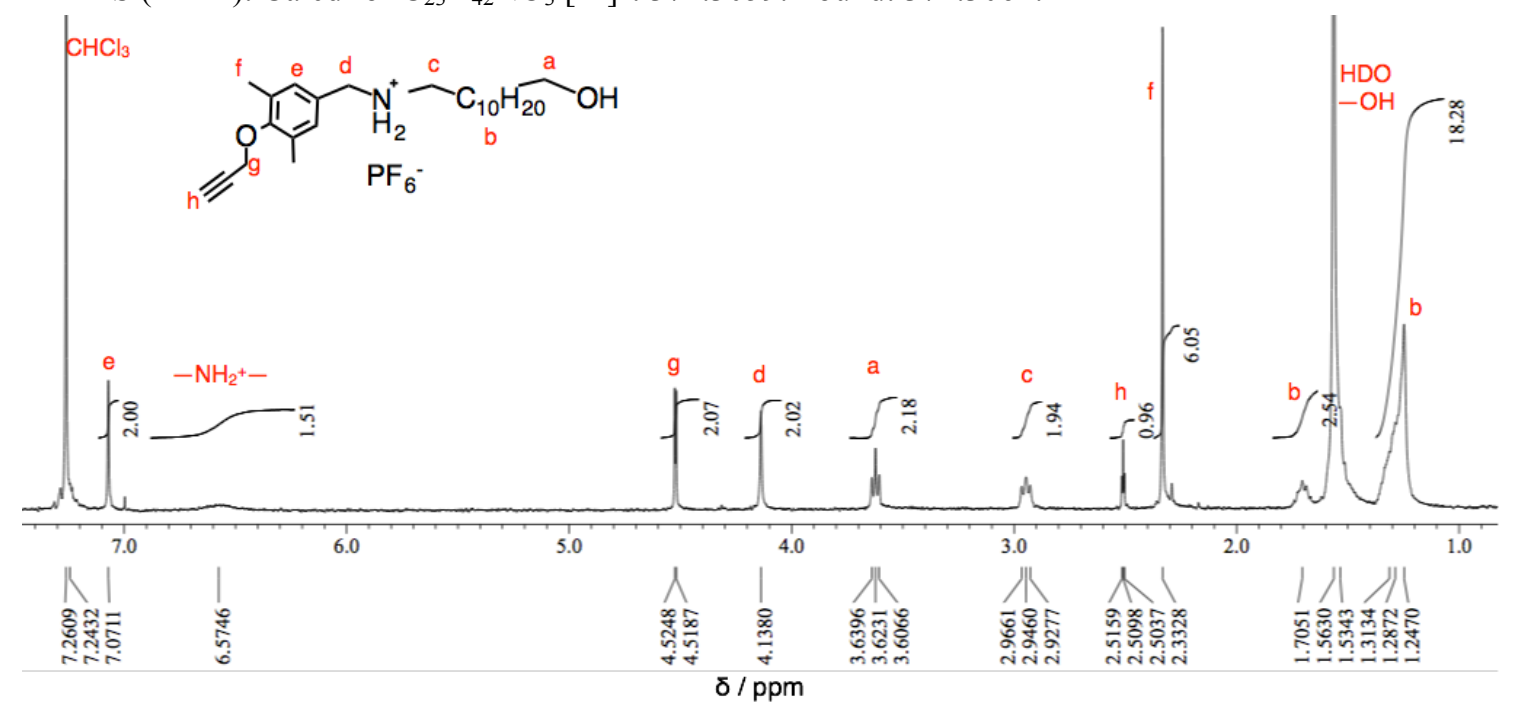

Figure S1. Assigned ${ }^{1} \mathrm{H}-\mathrm{NMR}$ spectrum of $1\left(400 \mathrm{MHz}, 298 \mathrm{~K}, \mathrm{CDCl}_{3}\right)$.

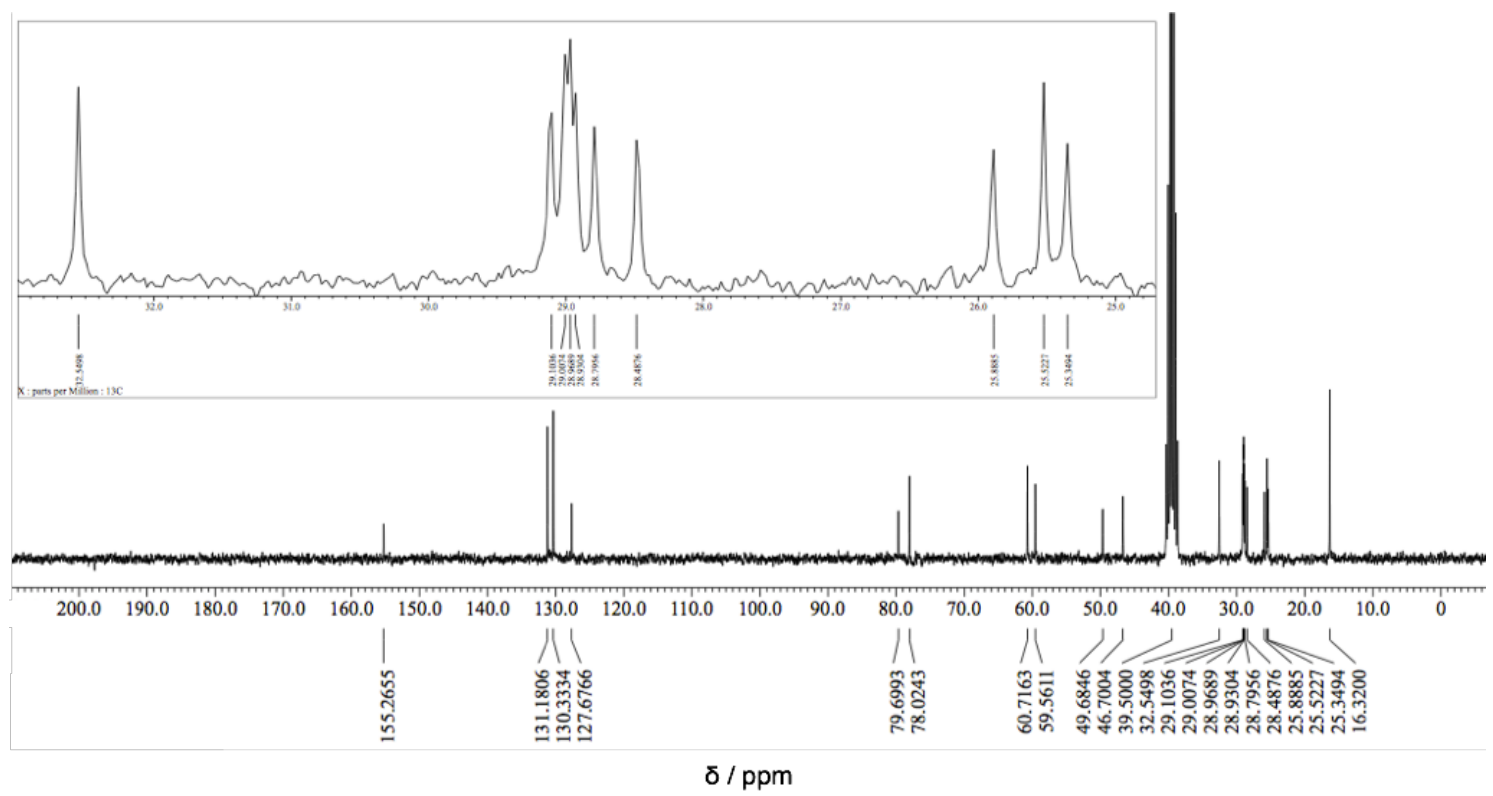

Figure S2. ${ }^{13} \mathrm{C}-\mathrm{NMR}$ spectrum of $1\left(75 \mathrm{MHz}, 298 \mathrm{~K}, \mathrm{DMSO}-d_{6}\right)$. 


\section{Synthesis of wheel component 2}

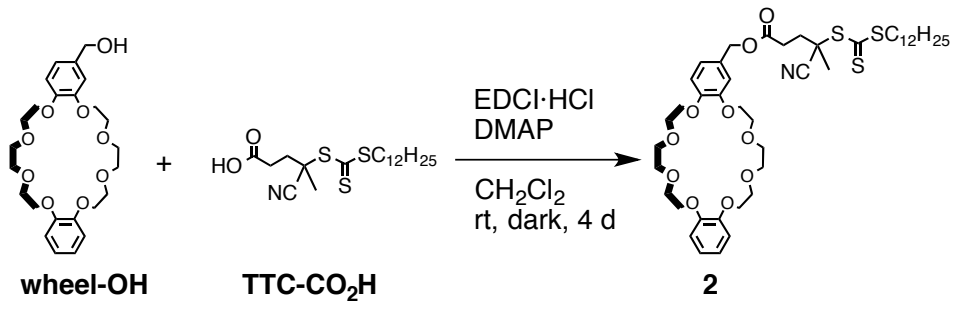

A solution of wheel-OH $(861 \mathrm{mg}, 1.8 \mathrm{mmol}), \mathbf{T T C}-\mathbf{C O}_{2} \mathbf{H}(811 \mathrm{mg}, 2.0 \mathrm{mmol}), \mathrm{EDCI} \cdot \mathrm{HCl}(386 \mathrm{mg}, 2.0$ mmol) and DMAP (22 $\mathrm{mg}, 0.18 \mathrm{mmol})$ in dichloromethane $(20 \mathrm{~mL})$ was stirred at ambient temperature under dark. After four days, $20 \mathrm{~mL}$ of $0.15 \mathrm{M} \mathrm{HCl}$ was added and the aqueous layer was extracted with chloroform three times. The combined organic layer was washed with $0.15 \mathrm{M} \mathrm{HCl}$, sat. $\mathrm{NaHCO}_{3}$ and brine, and dried over $\mathrm{MgSO}_{4}$, followed by evaporation to afford the crude product as yellow oil. Purification by preparative GPC yielded $2(1.29 \mathrm{~g}, 83 \%)$ as viscous yellow oil.

${ }^{1} \mathrm{H}$ NMR (300 MHz, $\left.298 \mathrm{~K}, \mathrm{CDCl}_{3}\right)$ : 6.94-6.80 (7H, m, aromatics), 5.03 (2H, s, a), $4.15(8 \mathrm{H}, \mathrm{m}, \alpha)$, $3.93(8 \mathrm{H}, \mathrm{m}, \beta), 3.84(8 \mathrm{H}, \mathrm{s}, \quad \gamma), 3.32(2 \mathrm{H}, \mathrm{t}, 7.6 \mathrm{~Hz}, \mathrm{~b}), 2.65(2 \mathrm{H}, \mathrm{t}, 7.6 \mathrm{~Hz}, \mathrm{c}), 1.86(3 \mathrm{H}, \mathrm{s}, \mathrm{d}), 1.7-1.2$ (22H, m, alkyl chain), $0.88(3 \mathrm{H}, \mathrm{t}, 6.7 \mathrm{~Hz}$, e (terminal Me)).

${ }^{13} \mathrm{C}$ NMR (75 MHz, $\left.298 \mathrm{~K}, \mathrm{CDCl}_{3}\right): 217.3,171.7,149.5,149.3,128.9,122.3,121.9,119.4,115.0,114.6$, 114.1, 71.6, 70.3, 70.2, 9.9, 69.9, 69.8, 67.3, 46.7, 37.5, 34.2, 32.3, 30.2, 30.0, 29.9, 29.8, 29.7, 29.6, 29.3, $28.1,25.1,23.1,14.5$.

HR-MS (ESI ${ }^{+}$): Calcd for $\mathrm{C}_{44} \mathrm{H}_{65} \mathrm{NNaO}_{10} \mathrm{~S}_{3}[\mathrm{M}+\mathrm{Na}]^{+}$: 886.3663 . Found: 886.3652

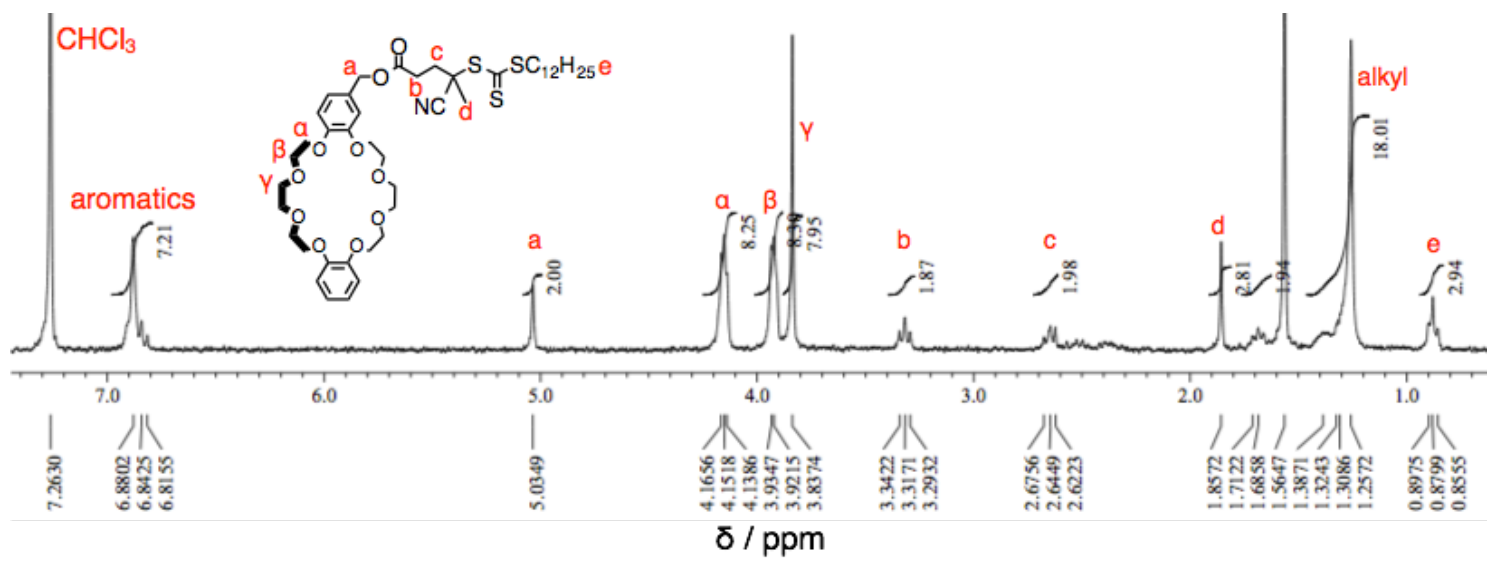

Figure S3. Assigned ${ }^{1} \mathrm{H}-\mathrm{NMR}$ spectrum of $2\left(300 \mathrm{MHz}, 298 \mathrm{~K}, \mathrm{CDCl}_{3}\right)$. 


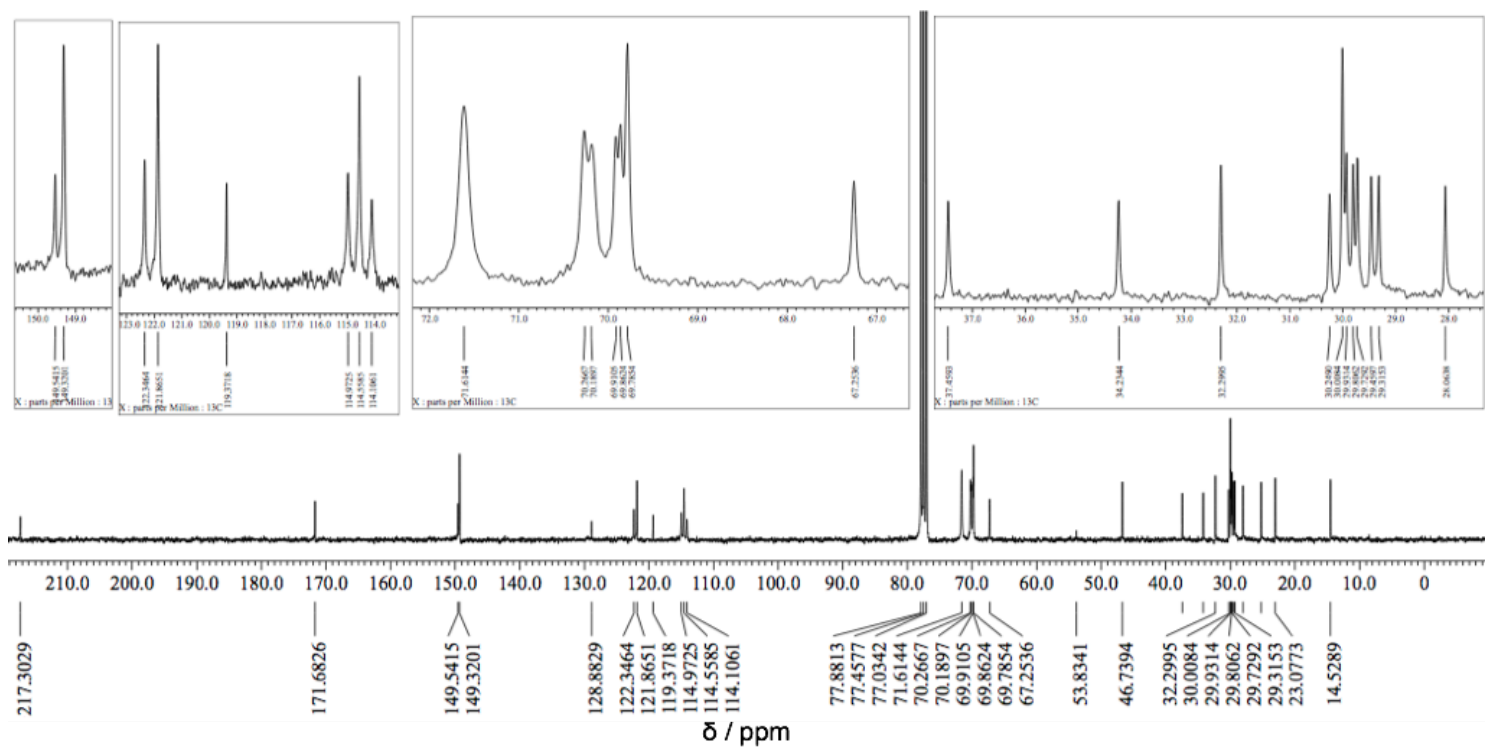

Figure S4. ${ }^{13} \mathrm{C}-\mathrm{NMR}$ spectrum of $2\left(75 \mathrm{MHz}, 298 \mathrm{~K}, \mathrm{CDCl}_{3}\right)$.

Synthesis of macromolecular [2] rotaxane $P V L_{x}$

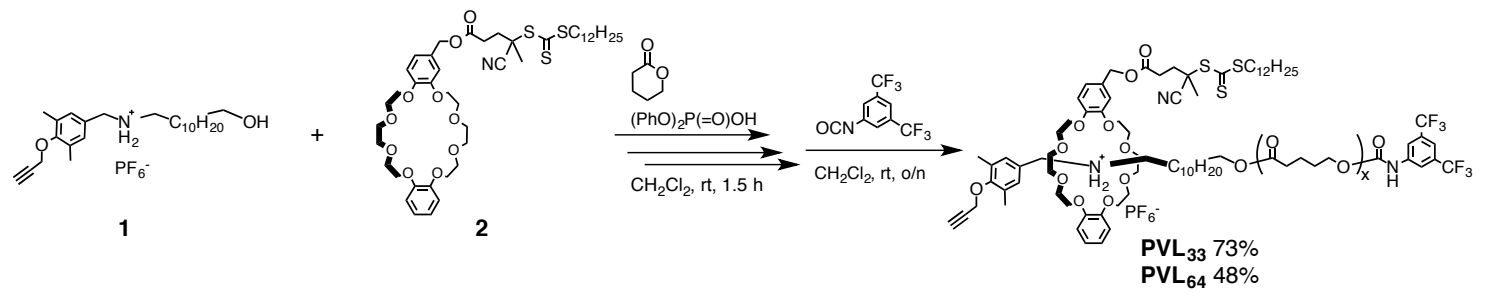

A mixture of the axle component $1(50 \mathrm{mg}, 0.096 \mathrm{mmol})$ and a wheel component 2 (90 $\mathrm{mg}, 0.10 \mathrm{mmol})$ in dry $\mathrm{CH}_{2} \mathrm{Cl}_{2}(1.6 \mathrm{~mL})$ was sonicated at ambient temperature until the solution got transparent to afford a solution of the pseudo[2]rotaxane initiator. Diphenyl phosphate (DPP) (25 mg, $0.10 \mathrm{mmol})$ and $\delta$-VL (0.29 $\mathrm{mL}, 3.2 \mathrm{mmol})$ were then added to the mixture under an argon atmosphere. After $1.5 \mathrm{~h}$, 3,5-bis(trifluoromethyl)phenyl isocyanate $(171 \mu \mathrm{L}, 1.0 \mathrm{mmol})$ was added to the resulting mixture and stirred overnight. The mixture was poured into a mixed solvent (ethanol/hexane $=1 / 9(\mathrm{v} / \mathrm{v})$ ) to obtain a crude macromolecular [2]rotaxane. Purification by preparative GPC yielded $\mathbf{P V L} \mathbf{3 3}(337 \mathrm{mg}, 73 \%)$ as a yellow solid.

The longer axle-tethering macromolecular [2] rotaxane $\mathbf{P V L}_{\mathbf{6 4}}$ (415 mg, 48\%) was prepared under similar conditions with 1 (56 mg, $0.11 \mathrm{mmol}), \delta$-VL (5500 mol\% vs 1) and $\mathrm{CH}_{2} \mathrm{Cl}_{2}(560 \mathrm{vol} \%$ vs $\delta$-VL).

Polymerization degrees of the $\mathbf{P V L}_{\mathbf{x}}$ were calculated by ${ }^{1} \mathrm{H}$ NMR using the integrals of signal 1 (5.03 ppm) and D (2.42-2.26 ppm).

${ }^{1} \mathrm{H}$ NMR (300 MHz, $\left.298 \mathrm{~K}, \mathrm{CDCl}_{3}\right): 7.99(2 \mathrm{H}, \mathrm{s}, \mathrm{b}), 7.72(1 \mathrm{H}, \mathrm{s}, \mathrm{c}), 7.53(1 \mathrm{H}, \mathrm{s}, \mathrm{a}), 7.23-6.80$ (9H, m, aromatics), $5.03(2 \mathrm{H}, \mathrm{s}, 1), 4.47(2 \mathrm{H}, \mathrm{m}, \mathrm{g}), 4.42(2 \mathrm{H}, \mathrm{d}, 2.4 \mathrm{~Hz}, \mathrm{j}), 4.26-4.00(\mathrm{~A}+\mathrm{d}+\alpha), 3.85(8 \mathrm{H}, \mathrm{m}, \beta)$, 


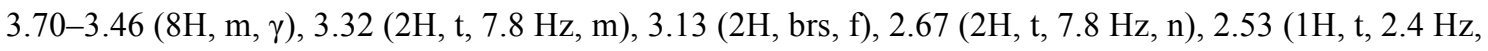
k), 2.42-2.26 (D), $2.13(6 \mathrm{H}, \mathrm{s}, \mathrm{i}), 1.88$ (3H, s, o), 1.78-1.56 (B+C), 1.35-1.00 (alkyl), 0.88 (3H, t, $7.2 \mathrm{~Hz}$, p).

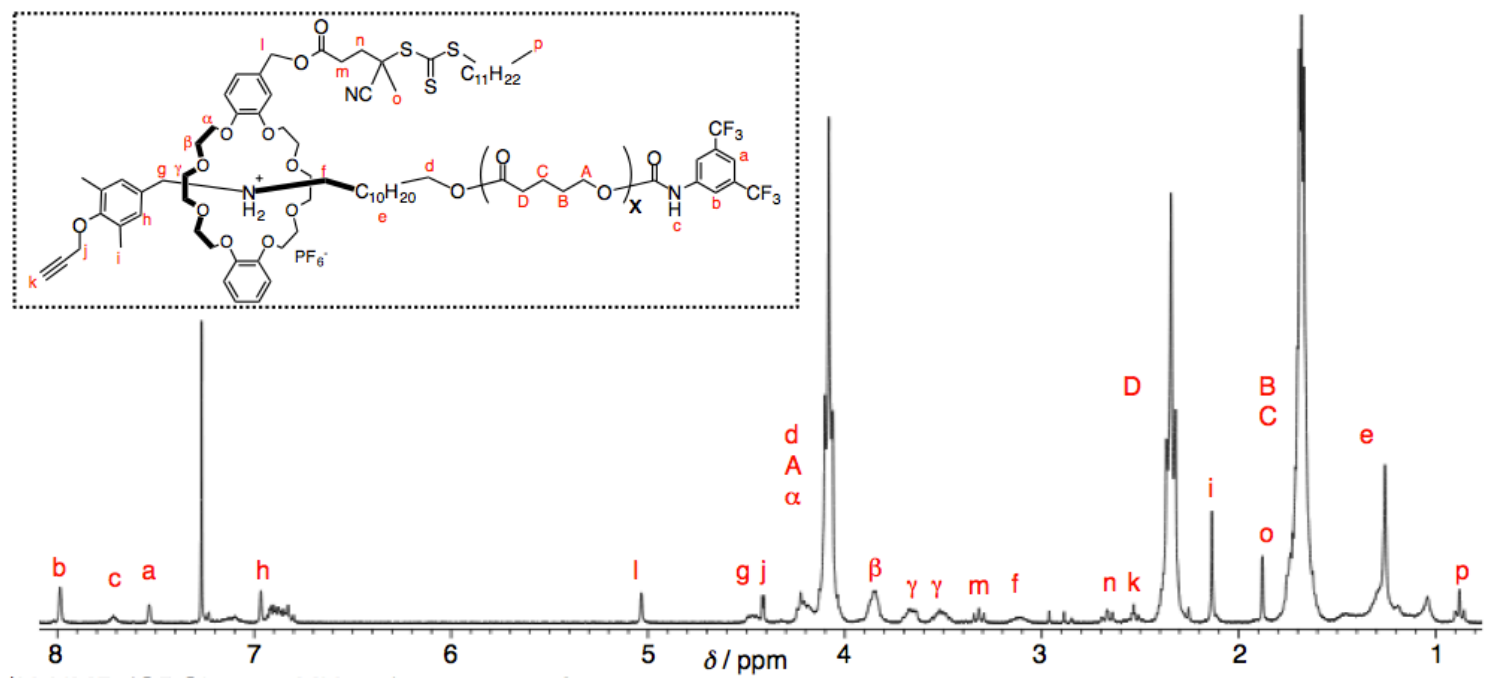

Figure S5. Assigned ${ }^{1} \mathrm{H}-\mathrm{NMR}$ spectrum of $\mathbf{P V L}_{33}\left(300 \mathrm{MHz}, 298 \mathrm{~K}, \mathrm{CDCl}_{3}\right)$.

Synthesis of rotaxane-linked diblock copolymer $\mathrm{PVL}_{\mathrm{x}}-$ rot- $\mathrm{PS}_{\mathrm{y}}$

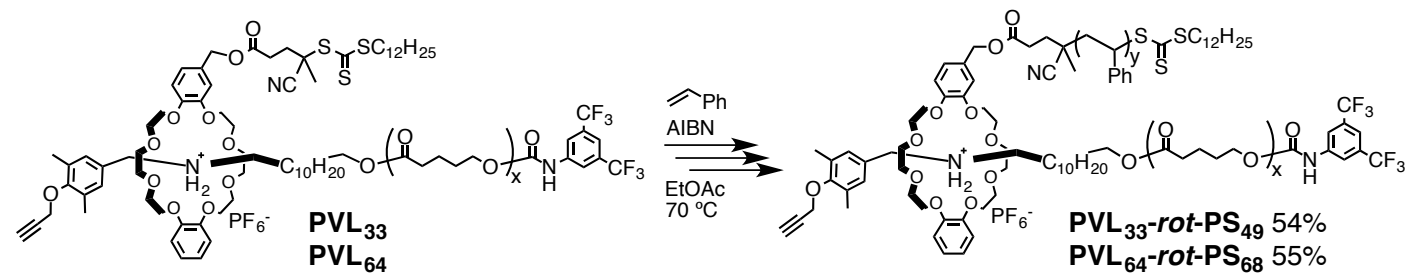

To a solution of macromolecular [2] rotaxane $\mathbf{P V L}_{33}(229 \mathrm{mg}, 0.050 \mathrm{mmol})$ in styrene ( $\left.858 \mu \mathrm{L}, 7.5 \mathrm{mmol}\right)$ was added $0.20 \mathrm{~mL}$ of a solution of AIBN in ethyl acetate $(13.1 \mathrm{mg}$ in $3 \mathrm{~mL}, 5.0 \mu \mathrm{mol})$ and degassed three times by freeze-pump-thaw cycles. Then the system was purged with argon, closed and stirred at $70{ }^{\circ} \mathrm{C}$. After $10 \mathrm{~h}$, the polymerization was quenched via rapid cooling in liquid nitrogen and exposure to air. The polymer was isolated by reprecipitation into methanol to afford the crude product as a yellow solid. Finally, reprecipitation into ether yielded $\mathbf{P V L}_{33}-$ rot-PS 49 (255 mg, 54\%) as a yellow solid.

The longer axle-tethering diblock copolymer $\mathbf{P V L}_{64}$-rot-PS $6 \mathbf{6 8}$ (402 mg, 55\%) was prepared under similar conditions with $\mathbf{P V L}_{64}$ (386 mg, $0.050 \mathrm{mmol}$ ).

Polymerization degrees of the $\mathbf{P V L}_{\mathbf{x}}$-rot-PS $\mathbf{P S}_{\mathbf{y}}$ were calculated by ${ }^{1} \mathrm{H}$ NMR using the integrals of aromatic signal (7.10-6.29 ppm) and signal of PVL (2.38-2.30 ppm).

${ }^{1} \mathrm{H}$ NMR (300 MHz, $298 \mathrm{~K}, \mathrm{CDCl}_{3}$ ): 7.98 (2H, s), 7.67 (1H, brs), 7.54 (1H, s), 7.10-6.29 (PS aromatics), 4.95 (2H, br), 4.39 (2H, br), 4.25-4.02 ( $\left.\mathrm{PVL}-\mathrm{COCH}_{2} \mathrm{CH}_{2} \mathrm{CH}_{2} \mathrm{CH}{ }_{2} \mathrm{O}-\right), 3.84(8 \mathrm{H}, \mathrm{br}), 3.70-3.46(8 \mathrm{H}$, 
m), $\quad 3.24(2 \mathrm{H}, \quad$ br $), \quad 3.11\left(2 \mathrm{H}, \quad\right.$ br, $\left.\quad \mathrm{ArCH}_{2} \mathrm{NH}_{2}{ }^{+} \mathrm{CH}_{2}-\right), \quad 2.50$ (1H, brs), 2.38-2.30 (PVL $\left.-\mathrm{COCH}_{2} \mathrm{CH}_{2} \mathrm{CH}_{2} \mathrm{CH}_{2} \mathrm{O}-\right), 2.12(6 \mathrm{H}$, brs), 2.0-1.2 (PVL+PS), 0.88 (3H, br).

\section{Synthesis of rotaxane-linked ABC star terpolymer $\mathrm{PEO}_{\mathrm{z}}-\boldsymbol{b}-\mathrm{PVL}_{\mathrm{x}}-$ rot $-\mathrm{PS}_{\mathrm{y}_{-}} \mathrm{A}$}

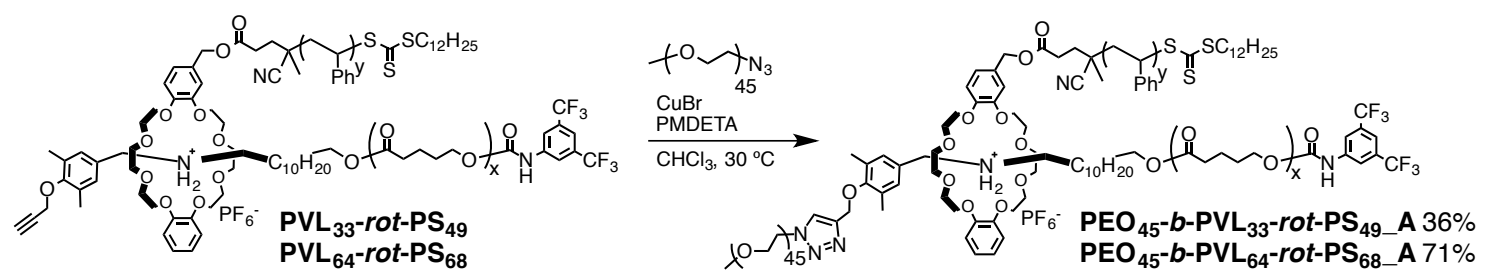

A solution of $\mathbf{P V L}_{33}$-rot-PS $\mathbf{4 9}$ (101 mg, $\left.0.010 \mathrm{mmol}\right)$ and azide-terminated PEO (Mw. 2000) (100 mg, $0.050 \mathrm{mmol})$ in chloroform $(1 \mathrm{~mL})$ was degassed twice by freeze-pump-thaw cycles. Copper(I) bromide $(8.8 \mathrm{mg}, 0.061 \mathrm{mmol})$ and PMDETA $(10.5 \mu \mathrm{L}, 0.050 \mathrm{mmol})$ were added under argon flow. The mixture was degassed twice by freeze-pump thaw cycles, purged with argon, closed and stirred at $30{ }^{\circ} \mathrm{C}$. The reaction was monitored by GPC. After 3 days, $0.15 \mathrm{M} \mathrm{HCl}$ was added and the aqueous layer was extracted with chloroform three times. The combined organic layer was washed with $0.15 \mathrm{M} \mathrm{HCl}$ and $\mathrm{NH}_{4} \mathrm{PF}_{6}$ aq. (60 mg in $20 \mathrm{~mL} \mathrm{H} \mathrm{H}_{2} \mathrm{O}$ ) in order, and dried over $\mathrm{MgSO}_{4}$, followed by evapolation to afford a crude polymer as a yellow solid. Purification by preparative GPC yielded $\mathbf{P E O} \mathbf{O}_{\mathbf{4 5}}-\boldsymbol{b}$-PVL $\mathbf{L}_{\mathbf{3 3}}$-rot-PS $\mathbf{P S}_{\mathbf{4 9}} \mathbf{A}$ (43 $\mathrm{mg}, 36 \%)$ as a reddish solid.

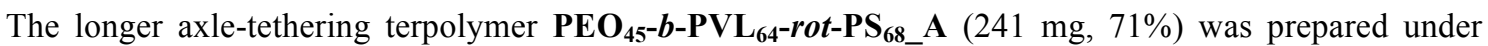
similar conditions with PVL $_{\mathbf{6 4}}-$ rot-PS $\mathbf{6 8}(301 \mathrm{mg}, 0.020 \mathrm{mmol})$.

${ }^{1} \mathrm{H}$ NMR (300 MHz, $\left.298 \mathrm{~K}, \mathrm{CDCl}_{3}\right): 7.98$ (2H, s), 7.72 (1H, brs), 7.53 (1H, s), 7.10-6.29 (PS aromatics), 4.25-4.02 (PVL - $\mathrm{COCH}_{2} \mathrm{CH}_{2} \mathrm{CH}_{2} \mathrm{CH}_{2} \mathrm{O}-$ ), 3.84 (8H, br), 3.70-3.46 (8H, m), 3.68-3.58 (PEO methylene), 3.38 (PEO terminal Me), 3.06 (2H, br, $\left.\operatorname{ArCH}_{2} \mathrm{NH}_{2}{ }^{+} \mathrm{CH}_{2}-\right), \quad 2.38-2.30$ (PVL $-\mathrm{COCH}_{2} \mathrm{CH}_{2} \mathrm{CH}_{2} \mathrm{CH}_{2} \mathrm{O}-$-), 2.14 (6H, brs), 2.0-1.2 (PVL+PS). 
Topology transformation of rotaxane-linked ABC terpolymer from star to linear

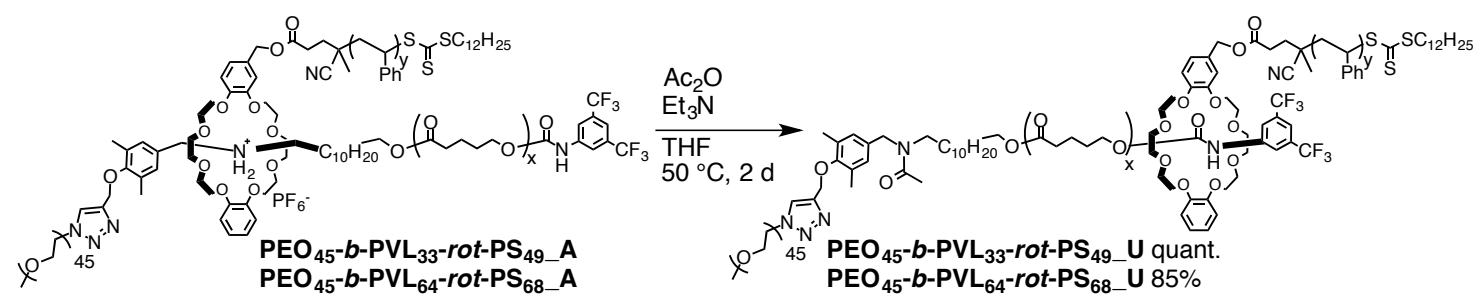

A solution of $\mathbf{P E O}_{45}-\boldsymbol{b}-\mathbf{P V L}_{33}$-rot-PS $\mathbf{P S}_{4} \_\mathbf{A}(32 \mathrm{mg}, 2.7 \mu \mathrm{mol})$, acetic anhydride $(16 \mathrm{mg}, 0.16 \mathrm{mmol})$ and triethylamine $(37 \mu \mathrm{L}, 0.27 \mathrm{mmol})$ in THF $(0.5 \mathrm{~mL})$ stirred at $50^{\circ} \mathrm{C}$ under argon atmosphere. After two days, the reaction was quenched by addition of water. Then, the mixture was extracted with chloroform four times, followed by passing through $\mathrm{MgSO}_{4}$ column and evaporated to afford a crude polymer as a reddish solid. Purification by preparative GPC followed by reprecipitation into cold hexane yielded $\mathbf{P E O}_{45}-\boldsymbol{b}-\mathbf{P V L}_{33}-$ rot $-\mathbf{P S}_{49} \mathbf{U} \mathbf{U}$ (38 mg, quant.) as a reddish solid.

The longer axle-tethering terpolymer $\mathbf{P E O}_{45}-\boldsymbol{b}$ - $\mathbf{P V L}_{64}-\boldsymbol{r o t}-\mathbf{P S}_{\mathbf{6 8}} \mathbf{U}$ (119 $\left.\mathrm{mg}, 85 \%\right)$ was prepared under similar conditions with $\mathbf{P E O}_{45}-\boldsymbol{b}$ - $\mathbf{P V L}_{64}-$ rot $-\mathbf{P S}_{68} \_\mathbf{A}(146 \mathrm{mg}, 8.6 \mu \mathrm{mol})$.

${ }^{1} \mathrm{H}$ NMR (300 MHz, $\left.298 \mathrm{~K}, \mathrm{CDCl}_{3}\right): 8.76$ (H, br, - NHCOAr), 7.89 (3H, br), 7.10-6.29 (PS aromatics), 4.25-4.02 ( $\mathrm{PVL}-\mathrm{COCH}_{2} \mathrm{CH}_{2} \mathrm{CH}_{2} \mathrm{CH}_{2} \mathrm{O}-$ ), 3.68-3.58 (PEO methylene), 3.38 (PEO terminal Me), 2.38-2.30 (PVL $-\mathrm{COCH}_{2} \mathrm{CH}_{2} \mathrm{CH}_{2} \mathrm{CH}_{2} \mathrm{O}-$ ), 2.0-1.2 (PVL+PS).

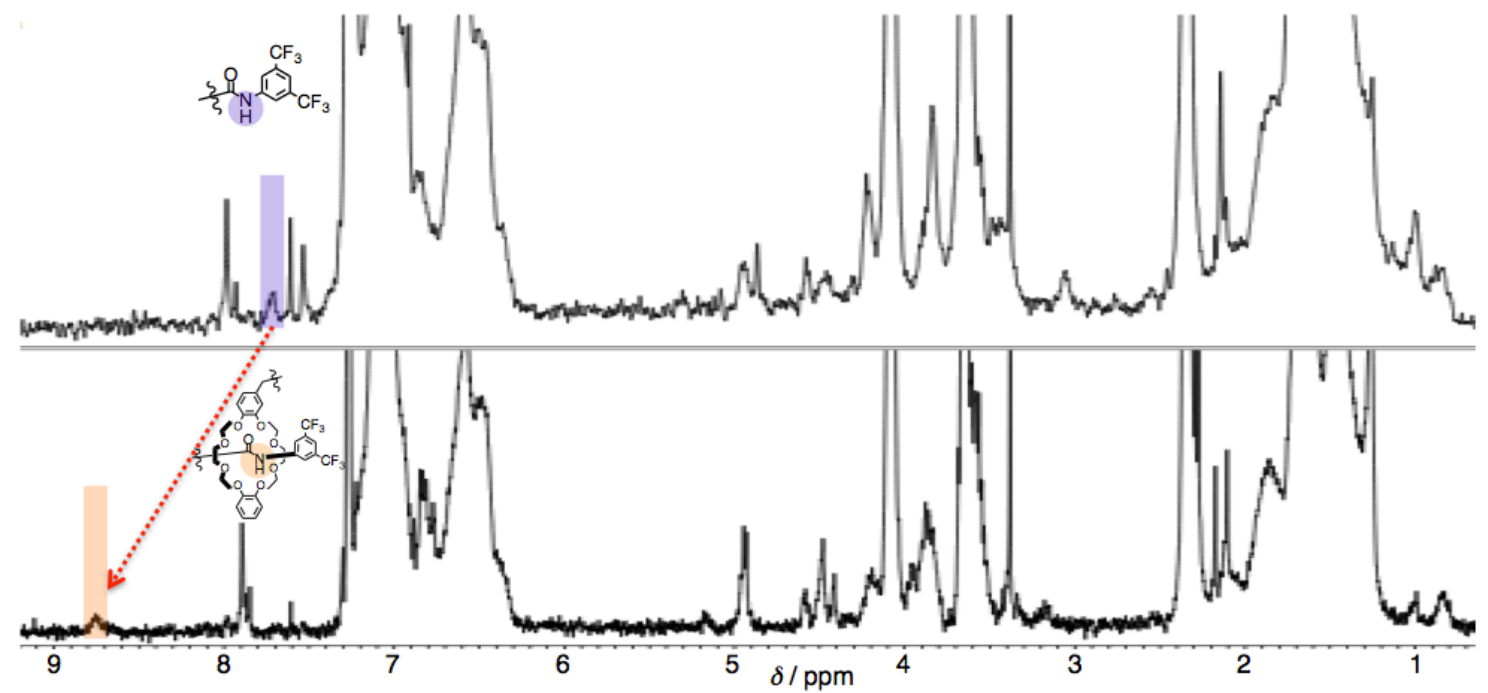

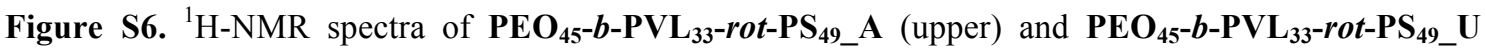
(lower) $\left(300 \mathrm{MHz}, 298 \mathrm{~K}, \mathrm{CDCl}_{3}\right)$. 

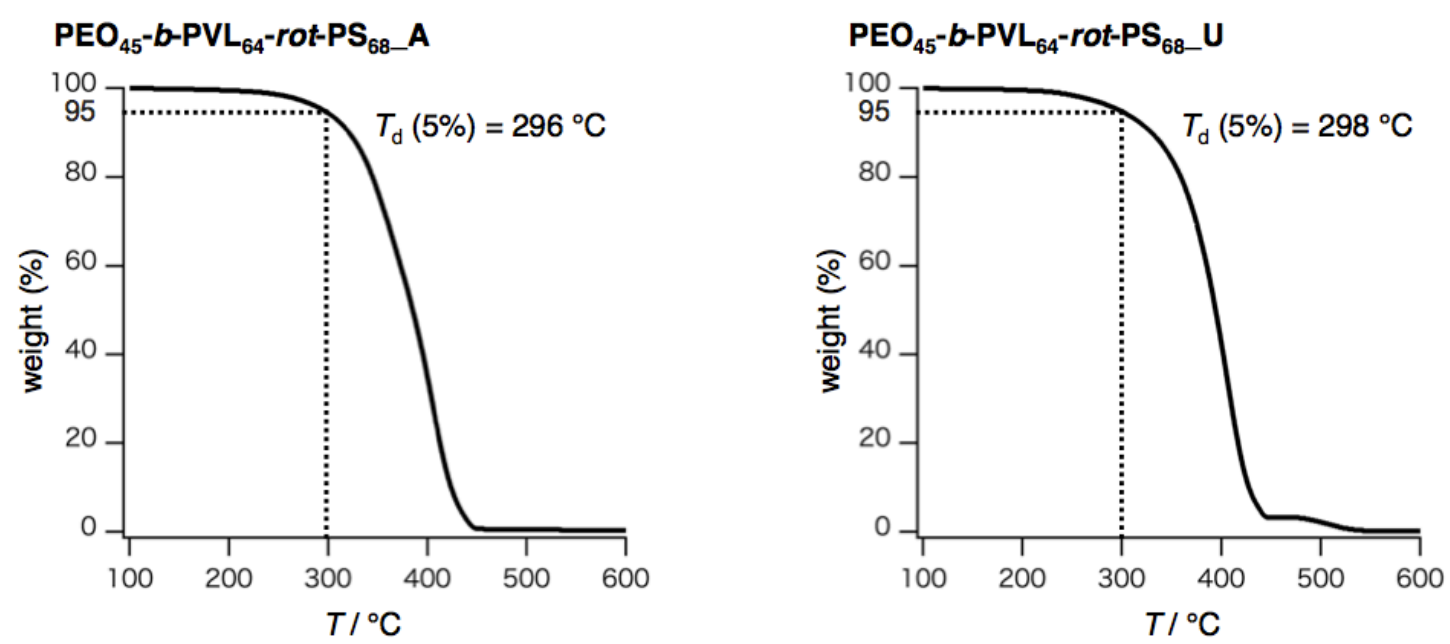

Figure S7. TGA curves of $\mathbf{P E O}_{45}-\boldsymbol{b}-\mathbf{P V L}_{64}-$ rot $-\mathbf{P S}_{68} \_\mathbf{A}$ (left) and $\mathbf{P E O}_{45}-\boldsymbol{b}-\mathbf{P V L}_{64}-$ rot $-\mathbf{P S}_{68} \mathbf{U}$ (right) (rate: $10^{\circ} \mathrm{C} / \mathrm{min}$ ).

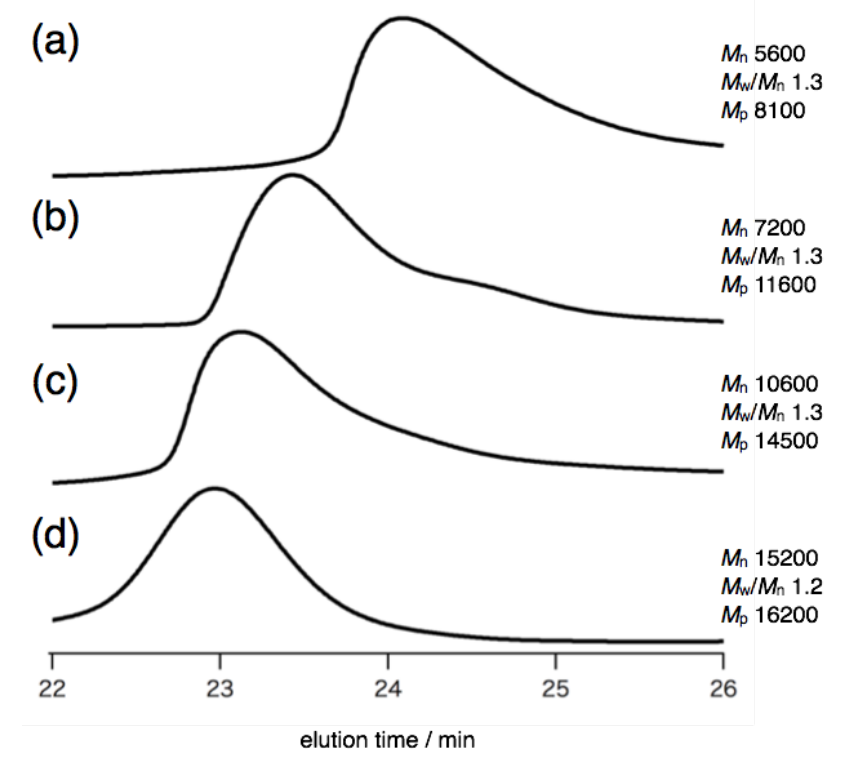

Figure S8. GPC traces of (a) $\mathbf{P V L}_{64}$ (b) $\mathbf{P V L}_{64}-$ rot- $\mathbf{P S}_{68}$ (c) $\mathbf{P E O}_{45}-\boldsymbol{b}-\mathbf{P V L}_{64}-$ rot $-\mathbf{P S}_{68} \mathbf{A}_{-}$(d) $\mathbf{P E O}_{45}-\boldsymbol{b}-\mathbf{P V L}_{64}-$ rot-PS $6 \mathbf{6 s}_{-} \mathbf{U}$ (eluent: $\mathrm{CHCl}_{3}$; detected by RI).

\section{References}

[1] Aoki, D.; Uchida, S.; Nakazono, K.; Koyama, Y.; Takata, T. ACS Macro Lett. 2013, 2, 461-465.

[2] Wang, F.; Han, C. Y.; He, C. L.; Zhou, Q. Z.; Zhang, J. Q.; Wang, C.; Li, N.; Huang, F. H. J. Am. Chem. Soc. 2008, 130, 11254-11255.

[3] Deng, J. J.; Luo, Y.; Zhang, L. M. Soft Matter 2011, 7, 5944-5947. 